\title{
Storage of Optical Information in Nano-size Cavity Arrays Under the Qubit-Light Interaction
}

\author{
Evgeny S. Sedov, Alexander P. Alodjants, Sergey M. Arakelian \\ Department of Physics and Applied Mathematics \\ Vladimir State University named after A. G. and N. G Stoletovs, Vladimir, Russia \\ alodjants@vlsu.ru
}

\begin{abstract}
We propose the model of one-dimensional polaritonic lattice representing the chain of weakly coupled optical nanocavities, each containing clusters of qubits. We revealed a variety of both localized and spreading dynamical regimes. We suggest a new physical algorithm for the spatially distributed storage of optical information where various localized dynamical states are used.
\end{abstract}

Keywords - polariton, optical information, qubit, soliton, spatially-periodic structure

\section{INTRODUCTION}

In the work we provide an alternative approach to optical information storage and retrieval by using half-matter, halfphoton property of coupled matter-light states, polaritons, in a one-dimensional (1D) qubit-cavity quantum electrodynamical (QED) array. We take into account the next-nearest photonic tunneling effects that become important because of small in comparison with optical wavelength cavity width. Both spreading and localized polaritonic states are revealed in the system. Transformation between matter-like and photon-like polariton solitons paves the way to the storage and retrieval of optical information through the adiabatic manipulation of detuning frequency [1].

\section{MODEl OF THE STRUCTURE AND PHYSICAL AlgORITHM FOR THE STORAGE OF OPTICAL INFORMATION}

We consider a 1D array of nano-scale cavities, each containing the ensemble of a small but macroscopic number of non-interacting qubits, see [2] and Fig. 1. Nearest and nextnearest cavities interact with each other due to overlapping of photonic wave functions. To analyze different regimes of polaritons in the cavity-QED arrays, we study the dynamical evolution of in-site Gaussian shape polariton wave-packet (PWP) in accordance with a variational principle.

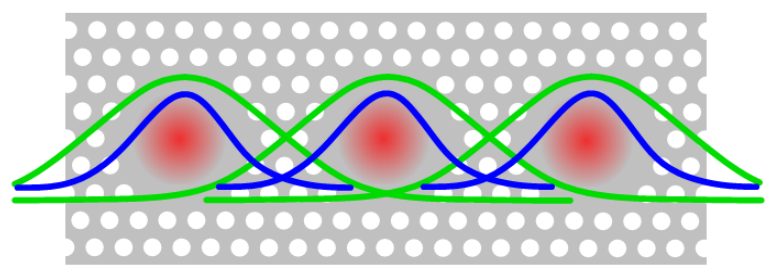

Fig. 1. Schematic for our proposed 1D cavity-QED array, in which each cavity contains the ensemble of two-level systems as qubits.

This work was supported by RFBR Grants No. 14-02-31443, and No. 14$02-97503$ and by the Russian Ministry of Education and Science state task 2014/13.

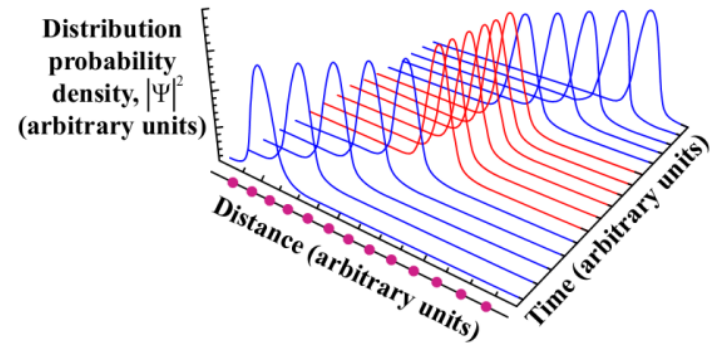

Fig. 2. Spatial-temporal evolution in a wave-packet storage and retrieval for the lattice polariton soliton wave packet.

In the system PWP exhibits four different dynamical regimes. First, it is a diffusive regime when PWP spreads in space. The second regime corresponds to a breathing regime when PWP width oscillates in time. Third, we deduce a selftrapping regime when a matter-like PWP can be stopped and localized within a few cavities. Last but not least one is regime of bright polariton soliton when PWP propagates with constant velocity and preserving its shape unchanged. The important peculiarity of the soliton which have been used to develop an algorithm for the spatially distributed storage of optical information is connected with the fact that it may be formed for both photon-like and matter-like polaritons - see Fig. 2.

At the first, writing, stage, a solitonic PWP enters the configuration of the cavity array; the polariton being photonlike. Then, by adiabatical switching of the matter-light detuning frequency, the photon-like lattice polariton soliton is transferred into a matter-like one with low group velocity. By reversing the detuning frequency, the original PWP can be reconstructed back to the photon-like polariton soliton at the output of the cavity array. The detuning frequency should be tuned with respect to the so-called rapid adiabatic passage (RAP) approach, which is slow on the time scale of inversed matter-field interaction and fast enough in comparison with any incoherent process occuring in the atom-light system.

\section{REFERENCES}

[1] E. S. Sedov, A. P. Alodjants, S. M. Arakelian, Y.-L. Chuang, Y.Y. Lin, W.-X. Yang, R.-K. Lee., "Tunneling-assisted optical information storage with lattice polariton solitons in cavity-QED arrays", Phys. Rev. A, vol. 89, pp. 033828, March 2014

[2] I-H. Chen, Y. Y. Lin, Y. - C . Lai, E. S. Sedov, A. P. Alodjants, S. M Arakelian, R.-K. Lee, "Solitons in cavity-QED arrays containing interacting qubits", Phys. Rev. A, vol. 86, pp. 023829, August 2012. 